\title{
How Do You Know When You Have Created A Bad Production?
}

\author{
James Simpson \\ 48 Silver Lane \\ West Wickham \\ Kent BR4 ORU \\ UK \\ James@jamessimpson3d.com
}

\begin{abstract}
Visualisation is a process and a tool that allows design teams to ideate, prototype, test and record a production design virtually. Theatre design is not a linear process and each production is different from another. Harnessing visualisation as a process and a tool enables designers to engage with each other and collaborate to produce strong, story driven designs which are more affordable, faster to make and of a higher quality.
\end{abstract}

Visualisation. Theatre. Opera. Ballet. Royal Opera House. Design thinking.

\section{INTRODUCTION}

The Royal Opera House has one of the busiest schedules of any theatre in the world and requires a design team to produce a full Opera or Ballet production in a fraction of the time a typical commercial producer would provide in the West End. Remedying this would mean decreasing performances or providing additional spaces for technical rehearsals, both of which have massive costs to the organisation.

In a perfect world, the solution would be to have a second Royal Opera House used solely for testing and producing our productions. What they have instead is a virtual second Opera House, complete in every detail with the same technical versatility that exists in real life. The $\mathrm{ROH}$ is constantly developing innovative visualisation tools in response to individual production requirements and has for a long time been the leader in the field of visualisation for the theatre.

The development and use of visualisation systems is prevalent in other design industries but in theatre and live events in general, the reason for using visualisation has often been reactionary. There are a number of reasons for this, but the two significant factors are; limited creative time which puts pressure on design teams and the increase in technological solutions being available to designers which require more complicated programming and therefore more technical time on stage to configure.
The term visualisation can be split into two categories; pre-visualisation and technical visualisation (Okun et al. 2015). Pre-visualisation represents the contextual, subjective view of a production that is discovered through a collaborative process of discussion and ideation, as opposed to technical visualisation, which is a methodical process of delivering data and information to create the most efficient use of stage time. Both these ideas will be discussed in subsequent chapters.

In the title, I have used the terms process and tools. It is impossible to talk about visualisation without addressing the technology and the systems that control it. These two terms work in symbioses with each other and can be talked about separately with the understanding that together they make up a complete visualisation system.

Although the Royal Opera House has ample evidence of visualisation systems and research, due to the complex nature of theatrical copyright this paper will not focus on specific user stories from this organisation and any names or references to real projects have been removed. The scope of the paper is not limited to Opera and Ballet but has been largely influenced by it and much of the analysis within is biased towards large-scale productions and may not be relevant to smaller productions. 


\section{VISUALISATION FOR OPERA AND BALLET}

Due to the significance of opening night in the theatre, it often feels as though no one wants to question the success of a production before it is presented in its final form. The danger in this is that many failures could be predicted early enough if they were more visible to the creative team and the producers.

Grant Hicks, theatre and film production designer expresses this perfectly in (Pavelka \& Chitty 2015):

As a theatre designer your credibility and integrity are a given and usually unquestioned; whereas in film it's questioned all the time.

Throughout the design process, there are clear milestones where the design is checked and reviewed by any number of experts. These milestones can be seen down to a granular level in everyday actions between artists and collaborators, but also on a macro scale, you can see key developments during phases of the production process.

Every production is different, even in a repertoire theatre with a repeatable business pattern, but as a general overview, these are theory milestones in creating a production:

- First contact between producer and director

- White card model

- Model presentation/design handover

- Performer rehearsals

- Technical rehearsals

- Dress rehearsals

- Previews (guest audience)

- Opening night

Within these major milestones are a multitude of deliverables, meetings, prototypes, research studies and creative discussions.

The point where we know we have a bad production is usually between previews and opening night. This is evident by the number of shows that close shortly after first night reviews versus shows that close during their preview period. It is the role of the preview period to allow design teams, often under pressure from producers, to make changes to the production design to account for feedback received from the preview audience. Having a preview audience is the most thorough form of visualisation before opening night it is possible to achieve, as every design element is available and in a mostly finished state, but even though the feedback is available to fix a broken show, it might be too late to make a meaningful change.
Interfering or modifying a designer's creative process undermines the trust you place in them by commissioning them to create the works in the first place. Sometimes designers are not working to the same goals as each other because the visual language each uses is different depending on their background and discipline. This makes communication weaker and therefore also collaboration. A strong director can often rectify divergences in the direction of the design, but for some directors, the divergence isn't visible to them without a stronger medium for communication.

The proposed solution here is to provide a collaborative communication tool that will help design teams to share their vision of the production through the use of visualisation systems.

\section{DESIGN THINKING IN THEATRE}

\subsection{Design thinking models}

Before the design can be fully implemented, there is the creative journey, which the design team goes on to develop and discover their production. It is an iterative process that relies on continual review, development, testing and decision-making.

There are many schools of thought on design thinking since first introduced by (Rowe 1987). The model that I prefer to use is that proposed by 5 Stages in the Design Thinking Process (Interaction Design Foundation n.d.), which uses five stages; Empathise, Define, Ideate, Prototype and Test.

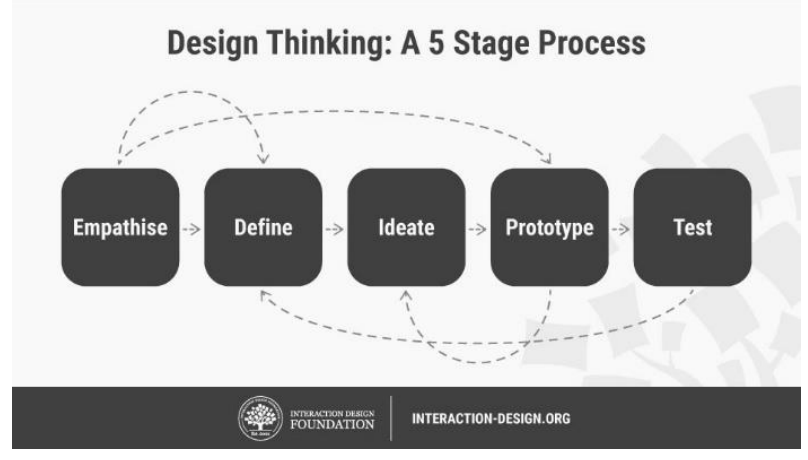

Figure 1: 5 Stages in the Design Thinking Process.

What is significant about this model over others is that it isn't a perfectly circular process and it recognises that you may need to return to ideation after your prototyping instead of moving forward to testing.

At each stage, there is a recognisable milestone in the development of a theatrical idea and it is clear to see how visualisation, in all its forms, supports particular stages: 
- Empathise is the research of the subject, understanding what the author intended or researching an issue or theme which the director wants to inject into their version of the story.

- Define is the establishment of the genre, the themes the director wants to portray, the message that they will send.

- Ideate is the creative discussions and brainstorming that gives rise to practical solutions to staging.

- Prototyping allows for an idea or solution to be made.

- Testing is the measured outcome of the prototype.

For theatre, I would suggest a sixth stage which sits after testing, and that is recording. It recognises when a design needs to be actioned into tangible and transferable data, such as a CAD drawing or saving lighting positions into a lighting console used to operate and control the virtual and real lights. This process, to a lesser or greater extent depending on the discipline, exists throughout the other five stages but ultimately must end with recording or it would be impossible to transfer a final idea to the real world.

My examples above cover the breadth of the production process but they can be considered at a granular level too, working for all elements of the creative process in between the major milestones.

\subsection{Design thinking in practical terms}

The significance of these stages to visualisation is that at the very least, Ideate, Prototype and Test can all be delivered through a visualisation system. Ideas will, in the first instance, occur through design discussion, but often there will be material, references, sketches or even a basic model box which would give designers the ability to ideate through a visual medium.

When a designer is ready to commit to $3 \mathrm{D}$, either card models or digital models, the ideation has already happened, partially through visual mediums that might include visualisation tools, and is ready to progress to testing.

To test something, first you need a prototype and in the physical world, this can be ignored if costs or time are short. Prototyping for all design disciplines is difficult because you don't see all the elements together at once until they are configured and working on stage. To test a complex visual lighting idea for instance you require every other visual element from scenery to costume available in order to see the complete visual picture of that lights performance in space.
There are specific examples where real-world testing can be achieved without needing much more than a few basic elements and this occurs occasionally in repertoire Opera through a process known as a Bauprobe (Carver \& White 2003). The Bauprobe is a technical visualisation planned well in advance of the set being built where mock-up scenic elements and lights are positions on stage to give a full-scale interpretation of the design for the artistic team to investigate.

Large engineering projects are also tested through complicated, expensive and risky temporary rigs, which allow a technical rigging solution or automation idea to be tested.

All of these solutions for testing are less practical and less timely than delivering the equivalent process digitally in a visualisation system. A digital prototype reduces the risk of danger from complex engineering prototypes, is cost effective as it only requires a $3 \mathrm{D}$ operator and a system, and timely as the operator can respond to ideas during the ideation stage (Carver \& White 2003).

Where current practice in most theatres is to take ideation through to testing using physical solutions, this may take several days or even weeks. The digital process can take minutes to hours, allowing a design team to iterate through their design thinking several times in a meeting on the same day.

\subsection{Design Iteration}

The challenge for design iteration in theatre is that it can't be achieved without good communication, which is an essential part of the collaborative design process. The design teams need to be in close proximity, on a regular basis, throughout the life of the project in order to make design decisions. However, the nature of theatre work means that designers are regularly travelling, entombed in a dark theatre or studio space for weeks or months for long days. It becomes very difficult to maintain regular communication on other projects, or even to focus on it when directly involved in the current project.

For true design iteration to work, each decision that is made will lead to a consequence for other disciplines in the project. Dealing with those consequences requires more decisions to be made which creates a waterfall effect of design changes. A truly iterative process is able to respond to these changes quickly so that the cycle of consequential design changes quickly reaches a compromise.

If creatives are not able to communicate effectively then this process cannot flow smoothly, and design changes require larger compromises, rushed 
changes or expensive last-minute alterations. The problem hasn't been identified early enough in the process to be incorporated into the technical delivery of the project.

In a later chapter on Virtual Reality, we will look at a solution to this problem which uses the power of immersive technology and social networking systems to deliver the flexible communication system required to support iterative design. For now, it is important context to pre-visualisation to understand that design iteration in theatre is hard to achieve because of the limited opportunities for communication available to design teams.

\subsection{Examples of design thinking in visualisation}

When a ballet company produced a production at an arena usually intended for live music, visualisation became an important instrument in identifying the cascading effects of each design decision.

The design was in a stable condition with everyone happy with the position of the scenery and the lighting relative to the stage and the audience. The orchestra was positioned behind the stage and on a platform behind a window that would allow the conductor to be seen by the audience throughout the performance. There was also masking in the wings to hide entrances and lighting positions carefully positioned to not be in the audience's view of the show.

During a design meeting hosted in a visualisation studio, the sightlines were being considered by moving the camera around the 3D model to see different seat views and assess the impact of their design.

It was noted that from certain high seats it was possible to see over the masking and into the wings and the quick-change areas, which is completely unacceptable. It is possible to identify the design iteration and design thinking that followed. This first observation is to appreciate that it isn't good for the audience to see the performers changing which is the empathise stage of design thinking process. Identifying the problem in your own project and asking the question "how else can we achieve good masking?" is define in the process.

Several ideas were then proposed by the assembled designers and production managers, which were sorted into priority. This stage is ideation. As the visualiser in charge of the system, it was my role to prototype these solutions in the 3D model which were then tested by checking the seat views from different locations. The results were then recorded for the production team to know what to commission the scenic carpenters to build. This exercise is an excellent example of design thinking in action in a theatrical production.

Now that a result has been achieved, the design iteration starts. The solution at this stage was to construct taller masking so that it would obscure the audiences view over the top. The impact of this is that now the lighting ladders which hang offstage of this position, are now unable to get a clear view of the stage. Another exercise in design thinking occurs and the conclusion is to raise the height of the ladders so they can see over the masking to reach the stage.

The consequence of this is that lighting trusses needed to hang above the ladders so the entire lighting rig had to be lifted up higher. This meant that the angle of incidence for the lights on the conductor was too acute and the conductor would be left in darkness. This led to the window for the conductor being raised up and the monitors for the orchestra being moved below the window instead of above which meant that the MD could no longer see the feet of the dancers when he turned around.

Through each consequence that was discovered, there was a design thinking process occurring, which led to a solution but caused another problem. Eventually, the cycle resolves itself by either discovering the perfect solution that solves all problems or by making a compromise to one area of the design. In this case, the solution went back to the masking itself and it was decided to build a roof over the original masking which meant everything stayed as it was. The original decision to make it higher was the best solution at the time but having been through an iterative design process the better solution for all design disciplines presented itself.

This exercise couldn't have happened without three things:

- The collective creativity and talent of the team.

- The availability of all of the design team who could be affected by a design decision to be in one place at the same time.

- A 3D visualiser that was able to demonstrate and prototype the solutions to problems in a fast and flexible way so that decisions can be responded to instantly.

The conclusion is that good design thinking and design iteration are essential to delivering a perfect product. Using a visualiser will enhance the process, making it more useful for design teams and allowing important decisions to be made quickly and confidently. 


\section{PRE-VISUALISATION}

The distinction between pre-visualisation and technical visualisation is that one focuses on the conceptual design and the other focuses on recording and planning tangible data to be transferred to the real world (Okun et al. 2015).

The benefits of pre-visualisation are hard to justify as it deals with intangibles and soft-skills that are less recognisable to producers. Nevertheless, the benefit of taking a design through a previsualisation process will exist, imperceptibly, in the final quality of the product.

At the beginning of the design process, when concepts are first being imagined, the design is very vulnerable and based on bold ideas that are untested. Designers work through the concept at this stage with sketches, drawings, model boxes and reference material. They are thinking about the story, the characters, how to deliver the narrative, what themes and issues are being presented and what sort of legacy do they want the production to leave.

Most of these ideas only exist cognitively as the design team search for inspiration to develop their idea. This is a very sensitive time when the design can be influenced for better or worse by external factors and introducing a visualiser at this stage could certainly be a factor for good or bad. Whilst it will help the design team to develop an idea, the suggestive visuals that are often created through assumptions by the modeller will lead them to a different design, possibly without them even realising it.

At this point in the process, the design is created for the first time as a white card model, which is a simple mock-up of the idea in a very flexible and easily modified card format, lacking textures and details. This is the first time, other than sketches and drawings that the design starts to be configured to represent a solid structure, but without consideration of technical constraint to allow complete creative freedom.

There are a lot of drawbacks to the white card model in the design process, not least that in an effort to remove technical considerations there is a reliance on the technicalities of constructing a stable, scaled cardboard model which has to contend with the same forces of physics that real scenery does but without the complex machinery and equipment that is used to defy those forces in a real theatre space.

There are also constraints to design changes in something that is physically built. Director Katie Mitchell describes "It is relatively easy for a designer to take large sections of the model apart and cut another bit of white card to make another door or a new wall for you to look at" (Mitchell 2009). This is less than ideal for design iteration if you need to return to an original idea or want to make further changes but can't because the model has been modified too often. It lacks the malleability of a digital model and the ability to track revisions and revert to them if needed. I would propose that working in 3D digitally at this stage would allow the design to retain its freedom and flexibility, which would help propagate the design process.

Another way pre-visualisation could be used is to bring the human factor into the 3D space by placing mannequins alongside the scenery and animating the movement of the scenery and people to see the complex positions and the relationship between all of the elements.

To summarise pre-visualisation, it is using $3 \mathrm{D}$ tools to support the creative process for the sake of creativity and not for functional planning. It should be used to facilitate the design process by creating a fertile ground for ideas to be generated.

\section{TECHNICAL VISUALISATION}

Tech-visualisation is a term adopted from the film and VFX industry as described in (Okun et al. 2015) where the term "fix it in post" can be heard often on set when a director or editor decides that something that could be fixed by re-filming in a studio would be easier left to be fixed by the postproduction team. The Third Floor, a visualisation company who has offices in London and LA have adopted a new term "fix it in pre". The intent is that a well-planned production won't have mistakes that need to be fixed.

This mentality was also taught to me by my school woodworking teacher with the phrase "measure twice, cut once". The intent again is that planning and checking will save you time and money by reducing mistakes.

Creative people aren't always drawn to technical solutions, relying on technicians to interface with it on their behalf, such as the lighting console operator, the fly-man or sound operator who all operate or configure technical equipment based on a designers direction. Therefore, advanced planning, which is usually conducted in $2 \mathrm{~d}$ drawing format, isn't an environment they feel comfortable working in for these types of decisions.

The lack of visual material isn't a problem with 3D visualisation tools, and with the advanced technologies that are now available, the director 
can get close enough to their design to be able to make design decisions early in the process. This doesn't preclude the ability to make changes in the real world, but many decisions that are taken in advance may have cost or time implications that can't easily be acted upon once the technical time on stage has begun (Carver \& White 2003).

A good use of technical visualisation is to engage the design team in a visualisation experience and make decisions that can be recorded to allow the technical delivery of the production.

Examples of technical visualisation may include:

- Lighting programming offline.

- Automation programming offline.

- Construction details (shapes, size, colours).

- Camera positions for filming and broadcast.

- Optimised lighting and sound positions.

- Sightline studies.

- Video programming and content creation.

We can put this into context with a real-life example. An Opera was trying to achieve the lighting through ceiling pieces that could tilt back and forth and fly up and down. Crucially, they also obscured the lighting rig and therefore the lighting had to come through three large holes built into random positions in the panels.

The lighting designer had clear positions on stage that needed lighting treatment. Using notes taken from rehearsals of the performers positions on stage, and comparing them with notes of the planned ceiling positions, it was possible to find the best place to hang a light on a bar above the hole in order to achieve the shot. To see if that same light could achieve a different location on stage the ceiling piece would move to a new scene and the position checked again.

As the study progressed, it was possible to optimise the position of the lights to satisfy the most regularly used space on stage. Records were kept of the orientation of the lights through the lighting console, which could play back the same position to a real light on stage, recreating the look. It also meant that a record was kept of the lights placement on the bar with the exact $X Y Z$ coordinate of its position so it could be hung that way on stage. This was critical because the light had to hang above the ceiling piece which meant there was no way to access it to make changes to its position once it had been flown into the air. This made the technical visualisation process key to the delivery of the production in real life, as it would have been impossible to iterate all of the options for the design without it.

\section{TOOLS AND TECHNOLOGY}

\subsection{Model boxes}

There are two types of model box: the white card and the final model. The white card model has been mentioned in section 4 and is essentially a first prototype of the design built to scale. The full model is a complete and accurate representation of the design that has been committed to. This model will be used as the reference for all construction, painting and crafting of the final scenery so every detail is essential. Designers use different mediums for model boxes from card, foam board, clay, metal, 3D printing, Lego bricks and on occasion, 3D digital models.

The choice of medium used is down to the designer and the process of making the model box is an essential part of the design process to allow the craft to form the way a designer wants. It would be wrong to presume that designers model boxes are an out of date practice, they are part of an individual's workflow and form the initial stages of design communication in the team as (Pavelka \& Chitty 2015) point out.

The next generation of designers will be trained in digital 3D skills that didn't exist or wasn't being taught even 15 years ago, so there is an expectation that designers model boxes will become more digital and less sculpted, painted or crafted model boxes will be seen. This is a natural evolution for design as skills and technology change, providing the designer is using the tools that work best for them it should remain a given that model boxes can exist in whichever medium works best for them.

The reason that model boxes are important to visualisation is that they represent the current standard practice for non-digital visualisation and the processes we use today in digital 3D were honed and developed with the use of physical model boxes. It is important to recognise that visualisation isn't exclusive to digital technology and that there are numerous analogue tools still in use that defined the processes now being adopted by digital.

\subsection{Standardisation}

One of the drawbacks of theatrical drawing and visualisation systems is the lack of continuity across the industry. Other industries such as architecture/construction, film, games and engineering have standards across their entire sector which encourages (if not demands) a set of rules for delivering information such as (Okun et al. 2015) VES handbook or (RIBA 2013). You could work for one studio and move to another and know 
where to find a specific drawing in a filing system, that naming conventions will be familiar and document codes allow multi-disciplinary practices to appear coordinated when the client receives multiple documents.

Introducing standards to the theatre would go a long way to improving the efficiency of document sharing and control, allowing us to share information easily and have fewer mistakes from information not being understood.

\subsection{Digital theatrical visualisation tools}

The focus of this paper has been on traditional 3D modelling systems used for CGI and construction. However, there are specific packages that are designed for the live events market and provide visualisation for specific disciplines.

There are at least five major producers of lighting visualisation software, which remains the most dominant form of technical visualisation in the theatre. This software requires a complete $3 D$ model of the design and using lights from the builtin library you can replicate your lighting rig with the virtual equivalent of any light on the market.

This program connects to the same lighting control console used to control the real lights and by listening to the UDP signal it sends can simulate real life lighting in the offline environment. The software operates with a refresh rate of $25 \mathrm{fps}$ allowing the lighting to appear real-time and lighting designers can plan and record all of their lighting positions virtually before transferring them to the stage.

\subsection{VR/AR/MR}

It would be impossible not to mention the latest innovation for visualisation technology: Virtual Reality (VR), Augmented Reality (AR) and Mixed Reality (MR), which collectively are referred to as Extended Reality (XR). It is now possible to create everything described in this paper using $X R$. Experiencing a design through $\mathrm{XR}$ has a powerful effect on the design team as they can see their production in full scale for the first time either in a personal experience in VR or a shared experience on location with $A R$ and MR.

Being able to walk around the venue and witnessing the design in full scale can provide insights and knowledge that previously would only have been possible when the set is finally built. It might force them to re-design their scenery, reblock the cast or change the layout on stage because they are able to connect the audience's point of view with their design and even introduce the human scale by having people walking around the visualisation experience.

XR has given theatrical visualisation a resurgence, where designers and directors are more interested in engaging because it removes the technical connotations of a monitor screen and 3D drawing.

\section{A vision for the future}

Almost all of what has been discussed in this paper has been achieved or prototyped. The technology is moving so fast that completed Research and Development is out of date before it is finished and a good use case scenario might come and go whilst waiting for the development of a prototype to finish.

The future of theatre visualisation will undoubtedly be the subject of future research. The vision, in brief, is to have a singular, unified visualisation engine, which can be accessed through multiple technology platforms at once. You can have a simultaneous experience with someone in Virtual Reality whilst you are in Augmented Reality but viewing the same scenic design and able to interact with each other's experience to share design ideas.

The visualisation itself would be a complete storyboard of the production with every creative discipline represented from music to scenery to performers, covering everything from the start of the overture to the end of the finale. This complete picture would provide the design team with the ability to produce a production in the flexible and controlled visualisation environment before being "transferred" to the stage for final design approval.

XR could be used in a number of ways. For instance, a director could join a networked design discussion from another country and their virtual presence could be replicated as an avatar for mixed reality users from the design team walking the real stage. Both teams would receive a complex and complete visualisation of the production and are able to interactively control and modify the production without needing much, if any, technical support from an operator. This idea is demonstrated nicely by Cirque de Soleil at the 2017 Microsoft Build Conference (Durbin 2017).

A similar system can be employed in the rehearsal room to provide technical facilities to a standard performance rehearsal. By connecting the stage control equipment (lighting desks, automation control, video equipment, etc.) to the visualisation experience, a production team and performers will be able to see the virtual scenery produced in front of them holographically. It will allow the performers to become aware of flying scenery, lighting positions, video projection cues and blackouts. It 
will also allow them to practice conducting scene changes in a safe and flexible environment where moving holographic scenery is done through gesture control and without the risks of physical scenery.

\section{CONCLUSION}

The title of this paper, "how do you know when you have made a bad production?" is a provocation designed to challenge our existing use of visualisation. There isn't a relevant answer to this question as it is supposed to highlight that the design process is a period of constant unknowns relying on calculations, good judgment, guessing and hope that the finished product will satisfy the audience and the producer.

Introducing 3D visualisation to the design process will provide confidence to the decisions being made and encourage creativity through design thinking and iteration.

Technology has a large role to play in the success of visualisation but it is the process that underpins it and has been relied on by design teams for centuries through traditional, analogue visualisation systems such as drawings and model boxes.

For the convenience of this paper, I have deliberately overlooked the fact that many productions work without visualisation support and great works of theatre can happen despite their lack of technology to help them create it. Productions like this work because of the talent of the creative team, the skills of the technicians who produce it and sometimes the luck of all of the pieces falling into place at the right time.

However, there is evidence in all design disciplines that a concerted and well-practised design process brings greater success to end products and the same can be true of the theatre. In these uncertain times when funding is being reduced to subsidised theatres and the costs of running a venue and producing shows have increased due to changes in the economy and the supply of tools and services, businesses look for an edge any way they can. Whilst some businesses might look to change their business model, there is clearly another more creative route, which can provide efficiencies and savings whilst supporting the design process. Visualisation is a tool for the creative teams, but one that can be appreciated by the producers as a way of improving their product for the audience and to save time and money whilst doing it.

\section{REFERENCES}

Carver, G. and White, C. A. (2003) Computer visualization for the theatre: $3 D$ modelling for designers. Elsevier/Focal Press.

Durbin, J. (2017) Microsoft Shows AR/VR Concept That Could Revolutionize How We Work. https://uploadvr.com/microsoft-vr-ar-workrevolutionize/ (retrieved 17 March 2018).

Interaction Design Foundation (n.d.) 5 Stages in the Design Thinking Process.

https://www.interaction-

design.org/literature/article/5-stages-in-the-designthinking-process (retrieved 19 February 2018).

Mitchell, K. (2009) The director's craft : a handbook for the theatre. Routledge.

Okun, J. A. et al. (2015) The VES handbook of visual effects : industry standard VFX practices and procedures. Second Edi. Focal Press.

Pavelka, M. and Chitty, A. (2015) So you want to be a theatre designer? Nick Hern Books.

RIBA (2013) RIBA Plan of Work 2013. https://www.ribaplanofwork.com/Default.aspx (retrieved 17 March 2018).

Rowe, P. G. (1987) Design thinking. MIT Press. 